\title{
The consumption of fruit juice rich in flavonoids increases plasma antioxidant status
}

\author{
Chutamat Niwat, Trevor George, Michael Gordon and Julie Lovegrove \\ University of Reading, Reading, UK
}

Fruits and vegetables rich in flavonoids have been related to the reduction in the incidence of CVD in several epidemiological studies ${ }^{(1)}$. Flavonoids are a group of phenolic compounds with strong antioxidant activity. Thus, the protective effects of the flavonoids could be a result of their ability to scavenge reactive oxygen and nitrogen species, which are formed during intracellular oxidative stress ${ }^{(2)}$. However, postprandial effects on antioxidant status after consumption of fruits and juices are controversial ${ }^{(3,4)}$. In addition, very little is known about the amount of flavonoids absorbed from the diet and the reliability of plasma biomarkers ${ }^{(5)}$. Consequently, the aim of the present study was to investigate the beneficial effects of an acute intervention with flavonoid-rich juices on markers of oxidative stress and cardiovascular risk factors.

A single-blind randomized postprandial study was performed in a group of twenty-four healthy free-living volunteers (twenty males and four females) with an age range of 28-64 years and a low habitual fruit and vegetable intake (less than five portions per d). Volunteers were asked to consume a low-flavonoid diet for $5 \mathrm{~d}$ before the study day and fast overnight the day before the study. The volunteers were randomly allocated to consume either $400 \mathrm{ml}$ flavonoid-rich juice (providing $768 \mathrm{mg}$ total phenolics (gallic acid equivalents (GAE)) including $142.2 \mu \mathrm{g}$ anthocyanins) or $400 \mathrm{ml}$ control juice, which had a low content of flavonoids (44 mg total phenolics (GAE)) but had the same sugar content. Two blood samples were taken at baseline and then fourteen subsequent samples at time intervals after the ingestion of the juice and throughout the study day for $8 \mathrm{~h}$. After an interval of 1 month they were asked to repeat the process with the second type of juice.

Plasma antioxidant status was measured before and up to $8 \mathrm{~h}$ after fruit-juice consumption by the ferric-reducing antioxidant potential (FRAP) assay ${ }^{(6)}$, the Trolox equivalent antioxidant capacity (TEAC) assay ${ }^{(7)}$ and the oxygen radical absorbance capacity (ORAC) assay ${ }^{(8)}$. Plasma vitamin $\mathrm{C}$, uric acid and total nitrates were also determined in the collected blood samples. It was observed that plasma FRAP increased significantly by $7.91 \%$ after ingestion of the flavonoid-rich juice compared with the flavonoid-poor juice $(P=0.031)$. This finding was confirmed by the TEAC and ORAC assays, which showed significant increases, by $8.89 \%(P=0.026)$ and $13.5 \%(P=0.015)$ respectively. In addition, plasma total nitrate and vitamin $\mathrm{C}$ increased significantly by $21.8 \%(P=0.001)$ and $34.9 \%(P=0.013)$ respectively. Correlations between these measures of antioxidant status and plasma levels of vitamin $\mathrm{C}$, uric acid and total nitrate were investigated. It was observed that only FRAP values correlated significantly and positively with plasma nitrate $\left(r^{2} 0.472, P=0.02\right)$. However, the correlation between plasma vitamin $\mathrm{C}$ and uric acid with antioxidant status will be re-investigated when data from all twenty-four volunteers are available.

It was concluded that ingestion of a flavonoid-rich fruit juice significantly increased postprandial antioxidant status in a group of healthy volunteers.

Funding provided by Unilever Bestfoods, The Royal Thai Government and University of Reading Endowment Trust Fund is gratefully acknowledged.

1. Halliwell B, Joseph R \& Jenner A (2005) Am J Clin Nutr 81, Suppl., 268S-276S.

2. Rohn S, Rawel HM \& Kroll J (2004) J Agric Food Chem 52, 4725-4729.

3. Dragsted LO, Pederson A, Hermetter A et al. (2004) Am J Clin Nutr 79, 1060-1072.

4. Mazza G, Kay CD, Cottrell T \& Holub BJ (2002) J Agri Food Chem 50, 7731-7737.

5. Erdman JW, Balentine D, Arab L et al. (2007) J Nutr 137, 718S-737S.

6. Benzie IFF \& Strain JJ (1996) Anal Biochem 239, 70-76.

7. Re R, Pellegrini N, Proteggente A, Pannala A, Yang M \& Rice-Evans C (2000) Free Rad Biol Med 26, 1231-1237.

8. Cao GH \& Prior RL (1999) Methods Enzymol 299, 50-62. 